\title{
Megakaryocytic alterations in thrombocytopenia: A bone marrow aspiration study
}

\author{
Pokharel $\mathrm{S}^{1}$, Upadhyaya $\mathrm{P}^{1}$, Karki $\mathrm{S}^{1}$, Paudyal $\mathrm{P}^{1}$, Pradhan $\mathrm{B}^{2}$, Poudel $\mathrm{P}^{3}$ \\ ${ }^{I}$ Department of Pathology, B.P. Koirala Institute of Health Sciences, Dharan, Nepal. \\ ${ }^{2}$ Department of Medicine, B.P. Koirala Institute of Health Sciences, Dharan, Nepal \\ ${ }^{3}$ Department of Pediatrics, B.P. Koirala Institute of Health Sciences, Dharan, Nepal.
}

\section{Keywords:}

Megakaryocytes;

Thrombocytopenia

\begin{abstract}
Background: Megakaryocyte morphology plays an important role in thrombopoiesis. A defect in any stage of megakaryocytopoiesis can lead to dysmegakaryocytopoiesis and thrombocytopenia. This study was conducted to understand megakaryocytic alterations and their contribution in the diagnosis of cases of thrombocytopenia.
\end{abstract}

Materials and methods: This was a cross-sectional study conducted on all consecutive cases of bone marrow aspirates of thrombocytopenia over a duration of one year in BPKIHS. Megakaryocyte morphology was studied with a 100X objective. Data were entered into Microsoft excel 10 and analysed with SPSS version 11.5. Descriptive statistics were charted and Chi-square tests were done for inferential statistics to find any association at 95\% Confidence Interval..

Results: Among the 38 subjects, megakaryocytic thrombocytopenia (44.7\%) was the most common cause of thrombocytopenia. Hypolobated megakaryocytes (63.2\%), bare megakaryocytic nuclei (57.9\%) were the common morphological changes in megakaryocytes. Odds of increased megakaryocyte count in megakaryocytic thrombocytopenia was found to be 12.5 times than for other causes of thrombocytopenia and the presence of bare megakaryocytic nuclei in Megakaryocytic Thrombocytopenia was statistically significant. ( $\mathrm{p}-$ value $<0.05)$

Conclusion: Many similarities were observed in megakaryocytic morphology among different hematological diseases. However, increased megakaryocyte count, and presence of bare megakaryocytic nuclei and hypolobated forms were significant in megakaryocytic thrombocytopenia.

\section{INTRODUCTION}

Megakaryocytes arise from pluripotent hematopoietic stem cells that undergo lineage commitment, proliferation and differentiation under the influence of cytokines, in particular thrombopoietin (TPO) to produce 1000-3000 platelets. ${ }^{1}$

\section{Correspondence:}

Dr. Saril Pokharel, MBBS, MD

Department of Pathology,

B.P. Koirala Institute of Health Sciences, Dharan, Nepal

Email:sairilp@gmail.com
It has become apparent that megakaryocyte morphology plays an important role in thrombopoiesis and that large megakaryocytes produce more platelets than smaller ones. The higher the ploidy (nuclear lobulation), the higher the platelet production.

The mechanisms that control platelet size are poorly understood but it seems that the body tries to maintain the total platelet mass and not the total platelet count. The total platelet mass is the constant derived when multiplying the 
total platelet count by the mean platelet volume. When the platelet count decreases, the mean platelet volume increases. When megathrombocytes (giant platelets) are seen on the peripheral smear it usually indicates peripheral consumption of platelets. Platelets play vital roles in repair of the minute vascular damages in day to day life, wound repair, the innate immune response and metastatic tumor cell biology. The average platelet count in humans ranges from $150-350 \times 10^{9} / \mathrm{L}$, although the level for any individual is maintained within fairly narrow limits.

Normal maturation and platelet formation results from megakaryocytic deoxyribonucleic acid (DNA) replication that occurs without cell division resulting in a large lobulated, polypoid nucleus. A wide variety of growth factors like thrombopoietin act synergistically with other hematopoietic cytokines and transcriptional factors stimulating the maturation and growth of megakaryocytes. A defect in any of the stages of megakaryocytopoiesis can lead to dysmegakaryocytopoiesis and thrombocytopenia. ${ }^{2}$

Thrombocytopenia is defined as platelet count less than $1,50,000 / \mathrm{mm}^{3}$. It is the most common cause of abnormal bleeding. Despite the number and diversity of disorders that may be associated etiologically, thrombocytopenia results from only four processes: Artifactual thrombocytopenia, deficient platelet production, accelerated platelet destruction, and abnormal distribution or pooling of the platelets within the body. ${ }^{3}$

Morphological changes in ITP (immune thrombocytopenic purpura) megakaryocytes, such as extensive cytoplasmic vacuolization, hypogranularity and smoothing of the cell membrane, were already described by Frank in 1915, and later confirmed by others. It was argued that these alterations were artifacts induced by fixation and/or staining methods. Similar abnormalities, however, were found using phasecontrast microscopy, by which cells can be examined in the living and unstained state. ${ }^{1}$

Dysplastic changes are well known in megakaryocytes in thrombocytopenia associated with myelodysplastic syndrome (MDS). However, they are also observed in megakaryocytes in non-myelodysplastic hematological conditions like immune thrombocytopenic purpura (ITP), infection associated thrombocytopenia (IAT), hypersplenism, aplastic anaemia (AA), acute myeloid leukemia (AML), acute lymphoblastic leukemia (ALL), leukemia lymphoma syndrome (LLS), bone marrow metastasis, blast crisis of chronic myeloid leukemia; but scant data exist on the prevalence of dysplastic changes in megakaryocytes in non-myelodysplastic hematological conditions. $^{2}$

A study done in 1995 showed that MDS is characterized by the presence of atypical micromegakaryocytes. ${ }^{4}$ Increase in number of megakaryocytes with immature forms were seen
Table 1: Distribution of Thrombocytopenia by causes

Causes of Thrombocytopenia

Frequency Percentage

\begin{tabular}{lcc}
\hline Megakaryocytic Thrombocytopenia & 17 & 44.7 \\
Acute Leukemia & 10 & 26.3 \\
Aplastic Anaemia & 5 & 13.2 \\
Megaloblastic Anaemia & 1 & 2.6 \\
Myelodysplastic Syndrome & 2 & 5.3 \\
Myelofibrosis & 1 & 2.6 \\
Mixed Nutritional Anaemia with & 2 & 5.3 \\
Megakaryocytic Thrombocytopenia & $\mathbf{3 8}$ & $\mathbf{1 0 0}$ \\
Total &
\end{tabular}

in Idiopathic Thrombocytopenic Purpura ( ITP) in $2009 .^{2}$ In 2010, a study showed megakaryocytes to be hyperlobated with fine stippled chromatin in megaloblastic anaemia. ${ }^{5}$ Similarly, a study done in 2012 concluded that immunemediated thrombocytopenic purpura (ITP) is characterized by significant morphologic alterations in megakaryocytes in the bone marrow. ${ }^{6}$

Further studies on the evaluation of megakaryocytic alteration and their contribution to thrombocytopenia can provide knowledge to the pathogenesis of numerous hematopoietic disorders that may identify broader clinical applications of the newer strategies to regulate platelet count and functioning.

This study was conducted with the objective to identify the alterations in number and morphology of megakaryocytes in different hematological disorders causing thrombocytopenia and evaluate if these alterations have any significant association with different causes of thrombocytopenia.

\section{MATERIALS AND METHODS}

This was a cross-sectional study conducted on all consecutive cases of bone marrow aspirates of thrombocytopenia over duration of one year in the Hematology Lab of BP Koirala Institute of Health Sciences, Dharan. The study frame included all the subjects with thrombocytopenia who had undergone a bone marrow aspiration in BPKIHS. Cases fulfilling the definition of thrombocytopenia but lacking marrow representative bone marrow in the marrow aspirate were excluded. Informed consent was obtained from the subjects and confidentiality maintained throughout the study. Ethical approval was taken from the Ethical Review Board, BPKIHS prior to the study.

The clinical details including history, physical findings, complete blood counts, peripheral smears and other relevant laboratory investigations required were noted. Peripheral smears were prepared and stained according to the guidelines in practical hematology using Jenner's Giemsa stain. ${ }^{7}$ In subjects with thrombocytopenia, bone marrow 
Table 2: Number of Megakaryocytes (Mk) in different causes of Thrombocytopenia

Number of Megakaryocytes per low power field (lpf)

Causes of Thrombocytopenia

Megakaryocytic Thrombocytopenia

Aplastic Anaemia

Acute Leukemia

Megaloblastic Anaemia

Myelodysplastic Syndrome

Myelofibrosis

Mixed Nutritional Anaemia with Megakaryocytic Thrombocytopenia

Total

\begin{tabular}{cccccc}
\multicolumn{2}{c}{$\begin{array}{c}\text { Normal } \\
(1 \mathrm{Mk} / 1 \text { to } 3 \mathrm{lpf}) *\end{array}$} & \multicolumn{2}{c}{$\begin{array}{c}\text { Increased } \\
(>2 \mathrm{Mk} / \mathrm{lpf})\end{array}$} & \multicolumn{2}{c}{$\begin{array}{c}\text { Decreased } \\
(1 \mathrm{Mk} / 5-10 \mathrm{lpf})\end{array}$} \\
Count & Percentage & Count & Percentage & Count & Percentage \\
2 & 28.55 & 15 & 83.3 & 0 & 0 \\
1 & 14.3 & 0 & 0 & 4 & 30.8 \\
1 & 14.3 & 0 & 0 & 9 & 69.2 \\
1 & 14.3 & 0 & 0 & 0 & 0 \\
2 & 28.55 & 0 & 0 & 0 & 0 \\
0 & 0 & 1 & 5.6 & 0 & 0 \\
0 & 0 & 2 & 11.1 & 0 & 0 \\
7 & $\mathbf{1 0 0}$ & $\mathbf{1 8}$ & $\mathbf{1 0 0}$ & $\mathbf{1 3}$ & $\mathbf{1 0 0}$
\end{tabular}

Table 3: Morphology of Megakaryocytes for different causes of Thrombocytopenia

\begin{tabular}{|c|c|c|c|c|c|c|c|c|}
\hline & MTP & $\mathbf{A A}$ & $\mathbf{A L}$ & MBA & MDS & MF & Mix MTP & Total \\
\hline Immature $\mathrm{MK}$ & $9(60.0 \%)$ & $1(6.7 \%)$ & $2(13.3 \%)$ & $0(0 \%)$ & $1(6.7 \%)$ & $0(0 \%)$ & $2(13.3 \%)$ & 15 \\
\hline Dysplastic MK & $1(33.3 \%)$ & $0(0 \%)$ & $1(33.3 \%)$ & $0(0 \%)$ & $0(0 \%)$ & $0(0 \%)$ & $1(33.3 \%)$ & 3 \\
\hline Bare MK nuclei & $14(63.6 \%)$ & $2(9.1 \%)$ & $1(4.5 \%)$ & $1(4.5 \%)$ & $1(4.5 \%)$ & $1(4.5 \%)$ & $2(9.1 \%)$ & 22 \\
\hline Emperipolesis & $1(33.3 \%)$ & $0(0 \%)$ & $1(33.3 \%)$ & $0(0 \%)$ & $1(33.3 \%)$ & $0(0 \%)$ & $0(0 \%)$ & 3 \\
\hline Platelet budding & $1(100 \%)$ & $0(0 \%)$ & $0(0 \%)$ & $0(0 \%)$ & $0(0 \%)$ & $0(0 \%)$ & $0(0 \%)$ & 1 \\
\hline Cytplasmic vacuolization & $1(33.3 \%)$ & $0(0 \%)$ & $0(0 \%)$ & $0(0 \%)$ & $2(66.7 \%)$ & $0(0 \%)$ & $0(0 \%)$ & 3 \\
\hline MicroMK & $1(100 \%)$ & $0(0 \%)$ & $0(0 \%)$ & $0(0 \%)$ & $0(0 \%)$ & $0(0 \%)$ & $0(0 \%)$ & 1 \\
\hline Hypo granular & $1(16.7 \%)$ & $1(16.7 \%)$ & $1(16.7 \%)$ & $0(0 \%)$ & $1(16.7 \%)$ & $0(0 \%)$ & $2(33.3 \%)$ & 6 \\
\hline Hypolobated MK & $15(62.5 \%)$ & $3(12.4 \%)$ & $1(4.2 \%)$ & $1(4.2 \%)$ & $1(4.2 \%)$ & $1(4.2 \%)$ & $2(8.3 \%)$ & 24 \\
\hline
\end{tabular}

*note: MK-megakaryocytes, MTP-Megakaryocytic Thrombocytopenia, AA-Aplastic Anaemia, AL-Acute Leukemia, MBA-Megaloblastic Anaemia, MDS-Myelodysplastic Syndrome, MF-Myelofibrosis, Mix MTP-Mixed Nutritional Anaemia with MTP.

was aspirated from posterior superior iliac spine, stained with Giemsa and examined under the light microscope (Olympus CH20i model).

The number of megakaryocytes was rated as ${ }^{1}$ :

Normal: 1 megakaryocyte per 1 to 3 low-power fields

Increased: > 2 megakaryocytes per low-power field, or

Decreased: 1 megakaryocyte per 5 to 10 low-power fields

Megakaryocyte morphology was studied with a $100 \mathrm{X}$ objective $^{8}$, which included nuclear segmentation, presence of immature forms, dysplastic forms, micromegakaryocytes, emperipolesis, platelet budding, cytoplasmic vacuolization, bare megakaryocytic nuclei, hypolobated forms and hypogranular forms. The presence of abnormal megakaryocytes which includes the micromegakaryocytes, dysplastic forms, megakaryocytes with separated lobes and hypogranular forms are considered as dysmegakaryopoiesis. Normal megakaryocytes are considered to have ${ }^{4-16}$ nuclear lobes. Immature megakaryocytes are defined as young forms of megakaryocytes with scant bluish cytoplasm and lacking lobulation of the nucleus which occupied most of the cell. Dysplastic megakaryocytes are defined as those with single/ multiple separate nuclei. Micromegakaryocytes are defined as megakaryocytes whose size is that of a large lymphocyte/ monocyte and which has a single/bilobed nucleus.

The megakaryocytes are considered to show platelet budding if there is budding of cytoplasmic processes from their surfaces. Hypogranular forms are defined as megakaryocytes with pale grey or water clear cytoplasm and sparse or no granules. The type of cell seen within the megakaryocyte in emperipolesis was also documented. The number and morphology of the megakaryocytes in thrombocytopenia was then assessed.

Collected data were checked for completeness and entered 
Table 4: Causes of Thrombocytopenia Versus Number of Megakaryocytes

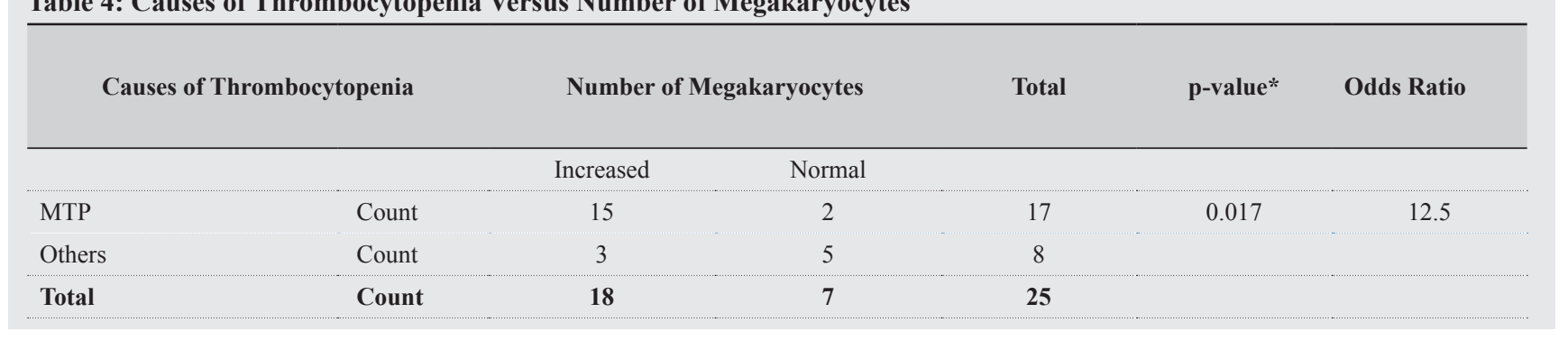

* Fischers Exact Test

Table 5: Bare megakaryocytic nuclei morphology versus causes of thrombocytopenia

\begin{tabular}{lccccc}
\hline \multicolumn{1}{r}{ Bare Megakaryocyte Nuclei } & Causes of Thrombocytopenia & Total & p-value* & & \\
& & & & & \\
& & Odds Ratio & \\
Present & Count & 14 & Others & 22 & 0.009 \\
Absent & Count & 3 & 8 & 16 & 7.5 \\
Total & Count & $\mathbf{1 7}$ & $\mathbf{2 1}$ & $\mathbf{3 8}$ &
\end{tabular}

* Fischers Exact Test

Table 6: Hypolobated form morphology versus Causes of Thrombocytopenia

\begin{tabular}{|c|c|c|c|c|c|c|}
\hline \multicolumn{2}{|r|}{ Hypo-lobated forms } & \multicolumn{2}{|c|}{ Causes of Thrombocytopenia } & \multirow[t]{2}{*}{ Total } & \multirow[t]{2}{*}{ p-value* } & \multirow[t]{2}{*}{ Odds Ratio } \\
\hline & & MTP & Others & & & \\
\hline Present & Count & 15 & 9 & 24 & 0.006 & 10 \\
\hline Absent & Count & 2 & 12 & 14 & & \\
\hline Total & Count & 17 & 21 & 38 & & \\
\hline
\end{tabular}

* Fischers Exact Test

into Microsoft excel 10 and analysed with SPSS version 11.5. Descriptive statistics in terms of frequency tables, pie charts, bar diagrams were prepared and Chi-square tests done for inferential statistics to find any association at $95 \%$ Confidence Interval.

\section{RESULTS}

Among the 38 bone marrow aspirate samples, thrombocytopenia was seen in all age groups though most subjects were below 20 years $(57.9 \%)$. Similarly females were seen to be slightly more affected $(57.9 \%)$. The most common clinical feature seen in case of thrombocytopenia was bleeding tendency $(\mathrm{n}=23 ; 60.5 \%)$ followed by pallor $(\mathrm{n}=2 ; 52.6 \%)$. In Acute Leukemia, organomegaly $(70 \%)$, fever $(80 \%)$ and pallor $(60 \%)$ were the common clinical features. Megakaryocytic Thrombocytopenia (44.7\%) was the commonest cause of thrombocytopenia for which bone marrow aspiration was sought followed by Acute Leukemia $(26.3 \%)$ as depicted in table 1 .

As shown in table 2, there was an increase in the number of megakaryocytes in 15 cases (88.3\%) of Megakaryocytic
Thrombocytopenia while there was a decrease in number of Megakaryocytes in 9 cases (69.2\%) of acute leukaemia. Looking at the morphology of megakaryocytes, a shift to young, immature, less polyploid megakaryocytes and bare megakaryocytes were the outstanding morphological feature noted in all the cases of Megakaryocytic Thrombocytopenia in the present study. Immature forms were seen in 9 cases $(60.0 \%)$, bare megakaryocytic nuclei in 14 cases $(70.0 \%)$, hypolobated in 15 cases $(62.5 \%)$ and micromegakaryocytes in one case of Megakaryocytic Thrombocytopenia. The morphology observed has been tabulated in table 3 .

Causes of Thrombocytopenia were categorized into Megakaryocytic Thrombocytopenia (MTP) and others due to the small sample size of the study and cross tabulated with the number of megakaryocytes to see if there was any statistical significance. Chi-square test was used to assess the association between variables with $95 \%$ confidence limits.

Since two cells had expected count less than five, Fisher's exact Test was used to calculate the significance of the association between different causes. The association was 
found to be highly significant with a $p$ value $<0.05$. The Odds of increased megakaryocyte count among MTP is 12.5 times than for other causes of thrombocytopenia $(\mathrm{OR}=12.5)$ as depicted in table 4.

The presence of bare megakaryocytic nuclei in Megakaryocytic Thrombocytopenia was highly significant (p- value < 0.05). The Odds of Megakaryocytic thrombocytopenia with bare megakaryocytic Nuclei was 7.5 times more than in other causes of thrombocytopenia as shown in table 5 .

The presence of hypolobated forms in Megakaryocytic Thrombocytopenia was highly significant with a p value of $<0.05$. (Table 6) The Odds of Megakaryocytic Thrombocytopenia with morphology of hypolobated forms was 10 times more than other causes of thrombocytopenia.

\section{DISCUSSION}

Thrombocytopenia, either persistent, isolated or in association with pancytopenia refractory to treatment is one of the commonly encountered hematological problems for which a bone marrow study is sought. The routinely prepared Giemsa stained bone marrow aspirate smears can help to observe the number and morphologic features of the megakaryocytes associated with different cases of thrombocytopenia. This can improve the diagnostic accuracy for a wide range of hematological disorders thereby enabling proper therapeutic interventions. ${ }^{2}$

In the present study, thrombocytopenia was seen in all ages with a minimum age of 3 years and maximum age of 74 years with mean age of 25.9 years. Out of total 38 cases, maximum number of cases (11 cases each, 28.95\%) were seen in less than 10 years age group as well as in 10-20 year age group. This was followed by 4 cases $(10.52 \%)$ each in $31-40$ and $41-50$ years age group. One case $(2.63 \%)$ was seen in age group of more than 70 years. Thrombocytopenia was more common in females $(\mathrm{n}=22 ; 57.9 \%)$ than in males $(\mathrm{n}=16 ; 42.1 \%)$. The commonest cause of thrombocytopenia for which bone marrow examination was sought was Megakaryocytic Thrombocytopenia. The second most common cause was Acute Leukemia followed by aplastic anaemia, megaloblastic anaemia and myelofibrosis were the least common.

In the study conducted by Muhury $\mathrm{M}$ et al, maximum number of cases (38/144 cases, 26.4\%) was seen in less than ten years of age group followed by $18.7 \%$ cases $(27 / 144$ cases) in 21-30 years of age. Least number of cases was seen in more than 61 years of age (9/144cases, $6.3 \%)$. Thrombocytopenia was commoner in males (24 cases, $63.2 \%$ ) than in females (14 cases, $36.8 \%$ ) in the first decade. ${ }^{2}$

The commonest cause of thrombocytopenia for which bone marrow examination was sought was AML (27/144 cases, $18.8 \%)$. The second most common cause was ITP (19/144 cases, $13.15 \%$ ) which was followed by ALL and dimorphic anemia $(18 / 144 \text { cases each, } 12.5 \%)^{2}$

In 15 of the 17 cases of Megakaryocytic Thrombocytopenia in our study, there was an increase in the number of megakaryocytes which was also observed by George et al , and Levine et al. They attributed this to stimulation of the marrow megakaryocytes to synthesize platelets at an increased rate due to immune-mediated platelet destruction in the spleen and other reticuloendothelial tissues. ${ }^{9} 10$

The severity of thrombocytopenia with increased megakaryocyotpoiesis, as observed by Wang ZY and Shen ZX, correlated with increased mean platelet volume (MPV) in patients with ITP. ${ }^{11}$

For MTP, increased megakaryocyte number was seen in $15 / 17(88.2 \%)$ and normal megakaryocyte number was seen in $2 / 17(11.8 \%)$ cases. For other causes of thrombocytopenia, increased megakaryocyte number was seen in $3 / 8$ cases (37.5\%) and those cases were of Mixed Nutritional Anaemia with MTP and MF. Normal megakaryocyte number was seen in 5/8(62.5\%) cases and those cases were of AA, AL, MDS, MBA. Fisher's exact Test was used to calculate the significance of the association between different causes - namely Megakaryocytic Thrombocytopenia Vs. Others (Aplastic Anaemia, Acute Leukemia, Megaloblastic Anaemia, Myelodysplastic Syndromes, Myelofibrosis and Mixed Nutritional Anaemia with Megakaryocytic Thrombocytopenia) and increased megakaryocyte number. The association was highly significant with a $p$ value $<0.05$. The Odds of increased megakaryocyte count among MTP is 12.5 times than for other causes of thrombocytopenia $(\mathrm{OR}=12.5)$. In a study on marrow megakaryocytes by Wang ZYand Shen ZX ${ }^{11}$, 38/70(54.3\%) cases had elevation of marrow megakaryocytes, and the number was within normal range in $24 / 70$ (34.3\%) cases, while $8 / 70(11.4 \%)$ cases displayed a number of megakaryocytes below the normal range. The anti-platelet antibodies in ITP have specificity for antigens associated with both platelets and megakaryocytes, so it is conceivable that the autoantibodies in ITP could react to megakaryocytes, leading to decrease of their number in marrow, making distinction between ITP with low megakaryocytes in marrow and amegakaryocytic thrombocytopenic purpura difficult. The conventional criterion in the diagnosis of ITP insisting that the number of megakaryocytes must be increased might be discussed and revised. For one investigator (Corrigan, 1988), the debate will be ongoing until there is a reliable method for separating hypo- from non-hypermegakaryocytic thrombocytopenic disorders. ${ }^{11}$

In 18 of the 19 cases of ITP, there was an increase in the number of bone marrow megakaryocytes in the study of Muhury $\mathrm{M}$ et al, ${ }^{2}$ similar to present study. The antiplatelet autoantibodies to glycoprotein IIb/IIIa and Ib/IX seen in ITP interfere with platelet production and release by causing megakaryocyte destruction and abnormal 
maturation. ${ }^{12-14}$ The destruction of megakaryocytes is either by phagocytic cells or by activation of complement or by induction of apoptosis. ${ }^{14}$ They also inhibit megakaryocyte colony formation and proplatelet formation, the defect being in the common erythroid-megakaryocyte stem cell. Therefore, altered megakaryocytic morphology and destruction resulting in defective platelet production and immune-mediated platelet destruction together contribute to thrombocytopenia. ${ }^{13}$ The fact that some patients respond to thrombopoietin mimetics further supports the fact that suppression of platelet production is an important mechanism in some ITP patients. ${ }^{14}$

In the study done by $\mathrm{Hu} \mathrm{T}$ et al, they observed overproliferation of bone marrow MKs in most ITP patients. The abnormality of MK might be one of the reasons for thrombocytopenia in some patients with ITP. ${ }^{15}$

In the study done by Dameshek $\mathrm{W}$ and Miller $\mathrm{E} \mathrm{B}^{16}$, in acute and chronic idiopathic thrombocytopenic purpura, although the platelets in the circulating blood were rare, megakaryocytes were increased in bone marrow, a finding similar to the present study. Platelet production was, however, greatly diminished and found in only 8 to 19 per cent of all megakaryocytes. Following splenectomy, there was a striking increase in platelet production, which was now present in 69 to 81 per cent of all cells; the large masses of new platelets in the marrow were often very striking.

The findings of increased megakaryocytes and greatly diminished platelet production in the marrow before splenectomy and the striking increase in platelet production after splenectomy indicate a definite pathogenetic relationship of the spleen to the disease. Idiopathic thrombocytopenic purpura is probably a form of hypersplenism (splenic thrombopenia) in which, through a possible hormonal mechanism, the megakaryocytes of the bone marrow are inhibited from normal platelet production and delivery. The marrow findings in idiopathic thrombocytopenic purpura are sufficiently characteristic to be of diagnostic value in differentiating the disease from leukemia and other conditions associated with a low blood platelet count. ${ }^{16}$ There are great similarities in morphological changes of megakaryocytes among different hematological diseases; however, the diagnostic approach will vary when detailed knowledge about morphological changes of megakaryocytes is available.

A shift to young, immature, less polypoid megakaryocytes and bare megakaryocytes was the outstanding morphological feature noted in most of the cases of MTP in the present study $(60 \%, 77.3 \%, 70 \%$ respectively). Similar findings were observed by Houwerzij et al, wherein they said it is because of apoptotic and para-apoptotic type of programmed cell death (PCD) of mature megakaryocytes. Most of the abnormal megakaryocytes were surrounded by neutrophils and macrophages, some in the state of phagocytosis. Inappropriate PCD of mature megakaryocytes can disrupt platelet formation and apoptosis-like PCD (para-apoptosis) occurs in ITP. This finding is especially useful when some patients of MDS present with isolated thrombocytopenia, thus mimicking ITP. ${ }^{1}$

Dysplastic forms, cytoplasmic vacuolization, platelet budding and emperipolesis was seen in 1 case $(33.3 \%)$ each and micromegakaryocytes in 1 case $(33.3 \%)$ of MTP in the present study. Emperipolesis, seen in 1 of the 17 cases of MTP $(33.3 \%)$ with lymphocyte in this study, was also observed in the study done by Roznan and Vives - Corrons. Their results show that megakaryocytic emperipolesis is a frequent event in different clinical conditions provided that a large number of megakaryocytes is examined, and with more than 200 megakaryocytes on the smear it can be demonstrated without exception. Consequently, they do not support the idea that the detection of megakaryocytic emperipolesis on bone marrow aspirate has any diagnostic significance. ${ }^{17}$

The cytoplasmic vacuolization seen in 1 case $(33.3 \%)$ which ultrastructurally represents mitochondrial swelling was also observed by Levine ${ }^{9}$ and Houwerzij et al, ${ }^{1}$ and this reflects an increased megakaryocyte turnover and indicates degenerative changes such as those of apoptosis and para-apoptosis. Another plausible explanation for the cytoplasmic vacuolization is autophagy to maintain cell metabolism when there is increased metabolic demand and nutrition deficiency due to increased megakaryocytopoiesis or it might be a way of sequestration and degradation of specific pathogens such as immunoglobulins. All the above features suggested dysmegakaryocytopoiesis which largely contributes to diminished platelet production.

The presence of bare megakaryocytic nuclei in Megakaryocytic Thrombocytopenia was highly significant with a $\mathrm{p}$ value $<0.05$. The Odds of Megakaryocytic Thrombocytopenia with bare megakaryocytic nuclei was 7.5 times more than in other causes of thrombocytopenia (namely, Aplastic Anaemia, Acute Leukemia, Megaloblastic Anaemia, Myelodysplastic Syndrome, Myelofibrosis and Mixed Nutritional Anaemia with Megakaryocytic Thrombocytopenia).

Bare megakaryocytic nuclei was seen in 16 cases of $\operatorname{ITP}(84.2 \%)$, their sensitivity being $84 \%$ and specificity $58 \%$ respectively in the study done by Muhury $\mathrm{M}$ et $\mathrm{al}^{2}$ .The association of presence of hypolobated forms in Megakaryocytic Thrombocytopenia was highly significant with a $\mathrm{p}$ value $<0.05$. The Odds of Megakaryocytic Thrombocytopenia with morphology of hypolobated forms was 10 times more than other causes of Thrombocytopenia (Aplastic Anaemia, Acute Leukemia, Megaloblastic Anaemia, Myelodysplastic Syndrome, Myelofibrosis and Mixed Nutritional Anaemia with Megakaryocytic Thrombocytopenia).

A shift to young, immature, less polyploid megakaryocytes 
and fewer mature platelet-producing megakaryocytes was the outstanding morphological feature noted in all the cases of ITP (sensitivity $=100 \%$, specificity $=68 \%$ ) in the study done by Manas Muhury $\mathrm{M}$ et al. ${ }^{2}$ Megakaryocytes were decreased or absent in aplastic anemia in this study which was also observed by Shadduck. They attributed this to bone marrow suppression and significant inhibition of nucleic acid synthesis in the megakaryocytes. ${ }^{18}$

In the study done by Dameshek W et al also, bone marrow aspiration results showed that the count of lymphocytes was increased and that of megakaryocytes was reduced in the bone marrow of the Aplastic anaemia patients, which showed failure of hematopoietic function. However, no morphological abnormalities appeared in bone marrow of Aplastic amenia. ${ }^{16}$ Whereas in the present study, immature, bare, hypogranular and hypolobated forms were detected.

The hypolobated, immature, bare and dysplastic forms along with emperipoliesis, cytoplasmic vacuolization and platelet budding of megakaryocytes were observed in the bone marrow aspirate slides of aplastic anaemis in the

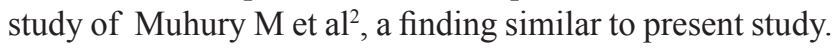
However, Tricot et al observed normal megakaryocytic morphology in aplastic anaemia. ${ }^{19}$

There were 10 cases $(13.2 \%)$ of acute leukemia in the present study and this was the second most common cause of thrombocytopenia for which bone marrow examination was sought. In 9 of the cases (64.3\%), the number of megakaryocytes was decreased with 1 case $(14.3 \%)$ showing normal megakaryocytes. Tricot et al, also reported decreased megakaryocytes in marrows of AML tightly packed with leukemic blast cells with maturation arrest.19 In the study done by Dameshek W et al, in acute leukemia, the megakaryocytes in marrow aspirations were conspicuously reduced, the few remaining cells present being of normal morphology. ${ }^{16}$

This study included only a few cases of MDS, Megaloblastic Anaemia, Myelofibrosis and Mixed Nutritional Anaemia with Megakaryocytic Thrombocytopenia for comparison. There were two cases of MDS, both of which showed normal megakaryocytes number in bone marrow. However, in the study done by Houwerij EJ et al, morphological studies of MDS bone marrow have shown increased numbers of abnormal megakaryocytes, in particular micromegakaryocytes with a low peak ploidy number (4$8 \mathrm{~N}$ ), suggesting an expansion of megakaryocytic precursors, an arrest in terminal megakaryocyte differentiation and impaired nuclear development. ${ }^{20}$ The increase in megakaryocytes which were present in 2 cases of Mixed Nutritional Anaemia showed that the patient had MTP who had subsequently developed Mixed Nutritional Anaemia .

In megaloblastic anemia, bare and hypolobated forms were seen. However, Wickramasinghe $\mathrm{SN}^{21}$ observed megakaryocytes with separation of nuclear lobes and nuclear fragments and attributed this to diminished DNA synthesis leading to nuclear maturation defect. The finding of emperipolesis in anemia was seen in the study of Tavassoli. ${ }^{22}$ However, emperipolesis and dysplastic megakaryocytes both were absent in the present study which could be due to a smaller sample size. ${ }^{21}$

Morphological changes of megakaryocytes in cases of thrombocytopenia due to myelodysplastic or non myelodysplastic causes are valuable and bring special interest especially in underdeveloped and developing countries due to the poor facitity for well sophisticated megakaryocyte studies like cultural studies, specific marker studies, electron microscope and ultrastructural studies.

This study shows that the diagnostic accuracy for different causes of thrombocytopenia will be improved by correlating different forms of megakaryocytes observed and their number present in bone marrow aspiration slide

\section{CONCLUSION}

There are many similarities in morphological changes of megakaryocytes among different hematological diseases; never the less, the diagnostic approach will vary when detailed knowledge about morphological changes of megakaryocytes is available. However, as seen in this study, increased megakaryocyte count, and presence of bare megakaryocytic nuclei and hypolobated forms were found to be significant in Megakaryocytic Thrombocytopenia. Understanding of the morphological changes of megakaryocytes in bone marrow aspirates can improve the diagnostic accuracy for a wide range of hematological disorders thereby enabling proper therapeutic interventions.

\section{Acknowledgements}

The author would like to acknowledge the contributions of entire staff of the Department of Pathology of BP Koirala Institute of Health Sciences and is also extremely grateful to Dr. Khem R. Sharma for his help in preparing the manuscript and Dr. Surya Niraula for his guidance in the statistical methods. Last but not the least the author would like to sincerely thank all the study subjects who gave their valuable time and effort despite their hardships to make this study possible.

\section{REFERENCES}

1. Houwerij EJ, Blom NR, van der Want JJ, et al. Ultrastructural study shows morphologic features of apoptosis and para-apoptosis in megakaryocytes from patients with idiopathic thrombocytopenic purpura. Blood 2004;103:500-6. Crossref

2. Muhury M, Mathai AM, Rai S, Naik R, Pai MR, Sinha R. Megakaryocytic alterations in thrombocytopenia: A bone marrow aspiration study. Indian J Pathol Microbiol 2009;52:490-4. Crossref

3. Greer JP, Foerster J, Rodgers GM et al. Wintrobe's Clinical Hematology.12th Edition. Philadelphia: Lippincott Williams and Wilkins;2009.Chapter 50, Thrombocytopenia: Pathophysiology and classification, $1291 \mathrm{pp}$. 
4. Ohshima K, Kikuchi M, Takeshita M. A megakaryocyte analysis of the bone marrow in patients with myelodysplastic syndrome, myeloproliferative disorder and allied disorders. J Pathol. 1995;177:181-9. Crossref

5. Bain B, Clark D, Wilkins B editors. Disorders of erythropoiesis, granulopoiesis and thrombopoiesis. In: Bone marrow pathology. 4th edn. UK: Willey Blackwell; 2010: p 472.

6. Deka L, Gupta S, Gupta R, Pant L, Kaur CJ, \& Singh S:Morphometric evaluation of megakaryocytes in bone marrow aspirates of immunemediated thrombocytopenic purpura. Platelets. 2012;1-5.

7. Lewis SM, Bain BJ, Bates I. Dacie and Lewis: Practical Haematology. Tenth edition. Churchill Livingstone, An Imprint of Elsevier; 2006. p166.

8. McKenzie SB, editor. Textbook of hematology. 2nd ed. Pennsylvania: Willaims and Wilkins; 1996. 2306pp

9. Levine FC. "Idiopathic" thrombocytopenia. Arch Intern Med 1999;88: 701- 28.

10. George JN, el-Harake MA, Raskob GE. Chronic idiopathic thrombocytopenic purpura. N Engl J Med 1994;331:1207-11. Crossref

11. Wang ZY, Shen ZX. Megakaryocytes and platelets in immune thrombocytopenic purpura. Baillieres Clin Haematol 1997;10:89107. Crossref

12. Wang L, Li Y, Hou M. Idiopathic thrombocytopenic purpura and dysmegakaryocytopoiesis. Crit Rev Oncol Hematol 2007;64:83-9. Crossref

13. McMillan R, Wang L, Tomer A, Nicho J, Pistello J. Suppression of in vitro megakaryocyte production by antiplatelet autoantibodies from adult patients with chronic ITP. Blood 2004;103:1364-9. Crossref
14. McMillan R. The pathogenesis of chronic immune thrombocytopenic purpura. Semin Hematol 2007;44:S3-S11. Crossref

15. Hu T, Shi XD, Feng YL, Liu R, Li JH, Chen J, Wang TY: Comparative study on bone marrow megakaryocytes in children with thrombocytopenic purpura, aplastic anemia and myelodysplastic syndrome.Zhonghua Er Ke Za Zhi.2005;43:183-7. Crossref

16. Dameshek W and Miller E B. The megakaryocytes in idiopathic thrombocytopenic purpura, a form of hypersplenism. Blood.1946;1:27-51.Crossref

17. Rozman C, Vives-Corrons JL. On the alleged diagnostic significance of megakaryocytic 'phagocytosis' (emperipolesis). Br J Haematol 1981;48:510. http://dx.doi.org/10.1111/j.1365-2141.1981.tb02745.x

18. Shadduck. Aplastic anemia: review of 27 cases. Lancet 2001;1:65767.

19. Tricot G, Vlietinck R, Boogaerts MA, et al. Prognostic factors in the myelodysplastic syndromes: importance of initial data on peripheral blood counts, bone marrow cytology, trephine biopsy and chromosomal analysis. Br J Haematol 1985;60:19-32. Crossref

20. Houwerij EJ, Blom NR, van der Want JJL, et al. Megakaryocytic dysfunction in myelodysplastic syndromes and idiopathic thrombocytopenic purpura is in part due to different forms of cell death. Leukemia 2006;20:1937-42. Crossref

21. Wickramasinghe SN. Morphology, biology and biochemistry of cobalamine- and folate- deficient bone marrow cells. Baillieres Clin Haematol 1995;8:441-59. Crossref

22. Tavassoli M. Modulation of megakaryocyte emperipolesis by phlebotomy: megakaryocytes as a component of marrow-blood barrier. Blood cells 1986;12:205-16. Crossref 